\title{
Compte rendu des travaux du Comité Technique de la Société Hydrotechnique de France
}

\section{Meetings of the Société Hydrotechnique de France}

\section{Session des 18 et 19 mars 1952}

\author{
English synopsis, p. 453.
}

Ont participé aux travaux de cette session:

a) Conseil d'adninistration :

M. Gariel, président.

mi. Bovghayer, Caguot, Cheviner, Henry, Hupnem, Mary, Nizery, de Rouvilis.

MM. Carrier et Legros représentant respectivement MM. Blanc et Coyne.

\section{b) Adhérents :}

Electricité de France représentée par MM. Amaz, Aunoy, Blanchet, Blet, Bonnet, Bonnin, Campuas, Cappus, Garinjer, Laqueille, Leconte, Loubaton, Pelletier, Rodier, Rogissart, Schneebeli, Talobre.

La Société Hydro-Electrique des Pyrénées, représentée par M. Hervé.

Les Ets Neyrpic, représentés par M. Casacci,

Le Bureau d'Etudes A. Coyne et J. Bellier, représenté par MM. LONDE et POST.

La Société des Tuyaux Bonna, représenlée par M. Cazals. La Société de Constructions Métalliques et d'Entreprises de Lyon, représentée par MM. Morne et Montamet.

La Société Dauphinoise d'Etudes et de Montages, représentée par M. Rambaud.

L'Entreprise Bachy, représentée par MM. Barmederte, Gutron et Leenhardis.

La Compagnie Fives-Lille.

La Société Générale d'Entreprises, représentée par M. Lebailuy.

Les Entreprises des Grands Travaux Hydrauliques, représentées par MM. Douves et Hamon.

L'Entreprise Léon Ballot, représentée par M. Smeveau.

Les Chaudronneries de Terrenoire, représentées par M. ARAud.

La Compagnie Industrielle de Travaux, représentée par M. Chautru et M. Le Chatelier.

La Société des Forges et Atelier's du Creusot, représentée par M. Forssat.
La Société de Constructions des Batignolles, représentée par M. Richardier.

L'Entreprise Ossude, représentée par M. Valleant.

c) Conité technique :

M. le Président Barmitron.

MM. Benoist, Bourgurgnon, Chamayou, Charron, Darrieus, Duffaut, Ferrandon, Fontaine, B. Gaspard, Gmert, Gridel, Haegelen, Mauser, Hénin, laflèche, Langlois, Leboux, Lescall, Méo, Montagie, Morlat, Pardé, Parmentier, PÉguY, de Santr-Vaulruy, Sanson, Sauvage de Saint-Marc, Serra, Silber, Suquet, 'Tenot, VARLET, VENNIN.

MM. Guiton et Founiser, représentant respectivement MM. Bergeron et Blosset.

\section{d) Invités :}

Lr Service Technique de l'Energie Electrique et des Grands Barrages, représenté par M. Melor.

L'Administration des Ponts et Chaussées, représentée par M. VASSEUR.

Le Laboratoire des Travaux Publies et du Bâtiment, représenté par M. HABIB.

L'Institut National de Statistiques, représenté par M. A. SANSON.

Ia Société Le Matériel Schneider-Westinghouse, représentée par M. Kennens.

La Compagnie Sulzer, représentée par MM. Gunfremor et PIENET.

La Société Rateau, représentée par M. Desmur.

La Société l'Energie Thermique des Mers, représentée par M. Ch. BeAU.

Mlle Garenc.

M. DE Beauchamp.

S'étaient excusés :

MM. Batycle, A. Borie, Caileux, Calvet, Coguand, Crescent, Fscande, Ferrand, Gaden, Gevthiat, Hermin. Mónard, MÉrier, Olivier-Martin, Sulzlée, Tricart, Vimert, les Fonderies Pignarre à Auxerre et la Société Hydro-Mécanique à Toulonse.

\section{SËANCE DU MARDI MATIN 18 MARS 1952}

La séance est ouverte à 9 h. 30 sous la présidence de M. Barrillon, Président du Comité Technique.

M. le Président communique les noms des membres ou personnalités qui se sont excusés de ne pouvoir participer aux travaux de la session.

\author{
Procès-verbal de la Session des 20, 21 \\ et 22 novembre 1951
}

Le procès-verbal, déposé au bureau, est mis à la disposition des personnes présentes. 


\section{Distinctions honorifiques}

M. le President est heureux de signaler an Comite Technique que $\mathrm{I}$. Albert Caquot, Administrateur de la: Société, et membre eminent de son Comité Technique, a été, d'une part, élu président à l'Académie des Sciences pour 1952 et, d’autre part, elevé à la dignité de grand. croix dans l'ordre de la Légion d'honneur.

M. le Président signale également les promotions an grade d'officiers de la Légion d'homneur de MM. Bacher el de Maublanc, membres du Comité Technique.

\section{Admissions au Comité Technique}

Le Comité approuve l'admission des personnalites ciaprès, présentées par M. Banniruon :

M. Allakd, Ingénieur hydrographe principal, membre du C.O.E.C.

M. Bablon, Directeur à la Société Alsthom-Charmilles.

M. LIEBER, Ingénieur en chef à la Société Alsthom-Charmilles.

M. Drouhrs, Chef du service de la Colonisation et de l'Hydraulique en Algérie.

M. Junet, Ingénieur des Travaux Publics de l'Etat.

\section{Démission}

M. Ie Président communique au Comité Technique la démission pour raison de santé de l'un de ses membres, M. Courrier, Ingénieur hydrographe en chef.

\section{Documents reçus depuis la dernière session}

a) Ouvrages francais :

M. Pande. - Crues méditertanćennes en septembre ei octobre 1933;

-. Inondations en France en 1935 et 1936;

-- Crue formidable dans le Roussillon, en octobre $19 ! 0$.

M. Pardé et DE Laboundonnale. - Les crues du Rhóne de novembre 19:4 à février 1945.

W. PARDE. - Rapport entre l'intensité des pluies et les débits maxima des crues.

- Etude critique sur la hauteur des crues

-.- Sur le mécanisme des transports solides effectués par les ribières. Citations d'auteurs et quelques formules.

- « Etudes hydrologiques ». Compte rendu de l'Annuaire Hydrologique de la France, 1949, et des Stations hydromériques francaises (Alpes). (Revue de Géographie de Lyon, $\mathrm{n}^{\circ} 4$, 1951);

- Les Chemins de fer des Elats-Unis.

-... La Bataille de Tannenberg.

M. Dunand-Dubres. - Pertes de charge dans les conduites (Rapport présenté à l'Association Internationale des Distributions deau, Paris, 1952).

M. Cardier. - Machines élévatoires. CCours de l'Ecole de Génie Rural.)

Laboratoire Dauphinois d'Hydraulique. - Thèse de M. McNown : «Sur l'entretien des oscillations des eaux portuaires sous l'action de la haute mer $\gg$.

René Dubnisay. - Les Procédés modernes d'épuration des eallx.

Ch. Maller et M. Gauthier. - Les Problèmes de l'eat en Algérie.

P. Tschaegle-Appent (Cie Générale des Eaux): La Pluviométrie de la région de Nice en 1950. La Pluviométrie de la région de Nice en 1951. La Plaviomélrie de 1893 à 1951. lues débits de la Vésubie (affluent du Var) de 1891 a 1951 .
M. Wmmax, - Elal des chutes de neige survenues pendant lhiver du $1^{\prime \prime}$ septembre 1950 an 31 mai 1951 pour le departement des llautes-dlpes.

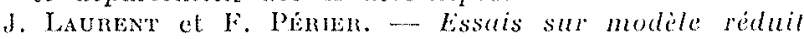
du port d'alger (envoi de M. Lannas).

A. Canllaux. - Les premiers enseignements glaciologiques des expedilions polates frangaises.

(Ce travail a été presenté le 6 juin à la sous-section de (ilaciologic.)

b) Ounages etrangers:

« Memori e Studi $\Rightarrow$ dell Istitulo di Idratiea del Politecnico di Milano:

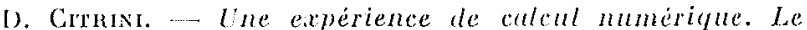
déversoir à plan circulaire $\left(n^{\circ} 87\right.$ ).

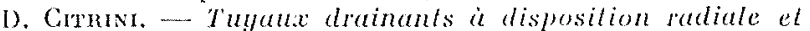
systène de puits dans une nappe artesienne (n" 88).

C. Fasso. - Sur ane intégrale qui s'est présentée dans une question d'hydraulique ( $\left.\mathrm{n}^{\circ} 89\right)$.

C. Fasso. - Sur le calcul hydraulque des conduites rectilignes élastiques (n० 90 ).

P.L. RomTa. - Le mowvement des fluides à travers les systèmes perméables ( $\left.\mathrm{n}^{\circ} 91\right)$.

Annuaire Hydrologique Allemand. - Bassin inféricur de l'Elbe, anné 1940, edité par Baubehorde Hamburgs Tiefbauamt Hauptabteilung Wasserwirtschaft Landesstelle für Gewässerkunde.

E.J. Devrovy. -.- Observations hydrographiques all Congo Belge et au Ruanda-Urundi (1948-1950).

D:. E. Reiches. - L'évaporation dans le cycle de l'eau sur la terre.

-... Energie électrique et Service météorologique.

-. Pas de changement dans les valeurs relatives des précipitations dans l'Allemagne du Sud.

Margareta Kender-HafFennegger. - Zur Hydrochemie des Grund' und Quellwassers in den Nördlichen Rheinlanden.

Dr. Jos. Van Emern. - Les variations des réserves naturelles d'eau de la rine gauche du Rhin inférieur au point de vue des eaux souterraines.

(Die Schwankungen des Naturlichen Wasserhaushaltes am Linken Niederrhein unter Besonderer Berücksichtigung der Grundwasserschwankungen).

L.J. Tison. - Recherches sur la tension limite dentratnement des matériaux contitutifs du lit.

Léon Lavin, - Mydraulique expérimentale.

M. Chavaz (Suisse). - Une machine à calculer le régime des lacs subjurassiens. (Communication du Service Fédéral des Eaux).

Bépot de la Bibliothèque Nationale. - Water Supply Papers du Geological Survey (E.U.A.) (années 1902 ì 1951).

\section{Pour information}

La Franckh'sche Verlagshandlung à Stuttgart nous a fait savoir (qu'elle tient a la disposition de nos membres des spécimens de la revue allemande d'hydraulique Die Wasserwirtschaft.

Les membres intéressés par eette annonce sont priés de s'inscrire at Secrétariat.

\section{Activité de la Société}

M. le Président porte à la connaissance du Comite T'echnique les travaux des differentes branches de la Société :

Révision alu Code d'essais des Installations hydrauliques. -- Le chapitre relatif aux mesures de débit par déversoirs a été examiné et mis an point par la Commission au cours des léunions des 29 novembre 1951 et 20 Prier 1952, sous la présidence de M. Escande.

Le bureat de la Commission a obtenu l'accord de 
divers auteurs de procédés de mesure des titrages et procéde à l'hamonisation de ces modalités d'essais qui seront annexées au nouveau Code.

Prochaine l'éunion courant arril sur les modalités d'essais des matchines hydrauliques.

Révision du cahier des charges des conduites forcées en métal. - Un avenant proposé relativement au calcul des conduites enrobées dans le rocher a été examiné par. la Commission au cours de la léunion du 22 février. Un nouveau texte en cours d'élaboration sera examiné ¿t une prochaine réunion.

Etude de la détermination des débits maxima des rues. - La Commission, réunie le 29 novembre 1951 sous la présidence de M. Barrulcon remplaçant M. Hupver sonffrant, a adopté un rapport de M. Montát sur les résullats des einq premières séances. Ce rapport est distribue aux membres de la Commission et pourra être communiqué aux personnes qui en feront la demande.

la Commission se réunira à nouveau lorsque son Burcau estimera qu'il y a lieu.

Etude du déficit d'écoulement. - Lá Commission crééc pour l'étude du déficit d'écoulement s'est réunie pour la première fois le 13 février. Un certain nombre de définitions ont été mises au point.

La Commssion, élargie par l'admission de quelques nouveaux membres, poursuivra ses travaux, au cours de la prochaine réunion sous la présidence de M. Senra. par l'étude de la « recherche d'une formule ».

Sous-section de Glaciologie. - Le 22 novembre 1951, la sous-section de Glaciologie a entendu la communication de M. Coutacine : « De la neige qui tombe à l'eau qui coule », présentée en séance plénière du Comits Technique, et assisté à la projection du film SnowHarvest.

Dans l'après-midi, au cours d'une deuxième séance de travail présidée par M. Chenrey, elle a entendu et discuté la communication de M. Chnors sur les « Observations faites dans les Hautes-Pyrénees pendant l'hiver 1950-51 sur les avalanches et les ouvrages paravalanches en vue de la protection de Barèges $»$, et le compte rendu des travaux de l'Association d'Hydrologie Scientifique à Bruxelles par M. Serra.

Des notes de M. VANNi sur la « Tournée glaciologique 1951 » et de M. Grinoux sur la schistosité du glacier de la Brenva ont été également examinées et commentées.

Section d'hydraulique fluviale et maritime. - Une nouvelle réunion de cette Section présidée le 21 janvier 1952 par M. DE Rovville a permis à ses membres d'entendre deux intéressants exposés : l'un de M. GouGENFEn sur « Un aperẹu sur les travaux récents concernant les lignes cotidales des océans $\gg$, l'autre de M. BrÉskr sur « l'Etude théorique de la propagation des houles par profondeurs lentement variables $\gg$.

Des discussions, auxquelles M. Mrche a pris une grande part, ont suivi ces exposés et, avant de se séparer, la section a jeté les bases de nouvelles et prochaines réunions.

\section{$2^{\text {mes }}$ Journées de l'Hydraulique (Grenoble, 1952)}

Il est rappelé que la prochaine réunion du Comité lechnique aura lieu à Grenoble du 25 au 29 juin 1952, dans le cadre des « $2^{\text {es }}$ Journées de l'Hydraulique », sur lo sujet : Transport hydraulique et décantation des matériaux solides.

Un aperçu est donné des travaux présentés et des visites et excursions organisées.

\section{Prix Henri-Milon 1952}

Le concours sera clos le $1^{\text {or }}$ avril 1952. Prière est faite aux ateurs-candidats do remette leurs travaux avant cette date.

\section{Séance spéciale du 21 mars 1952}

La S.H.F. organise le 21 mars, a 16 h. 30, salle Marcoau-Chaillot, une séance spéciale destinée a montrer aux présidents et directeurs générax des Societés adhérentes et de celles qui sont susceptibles de nous apporter leur concours à l'avenir, l'utilité industrielle et économique des sciences et techniques faisant l'objet de nos activites.

(On trouvera dans ee numéro un comple rendu de cette séance et les exposés qui y ont été faits.)

\section{Publications de la Société}

a) Parues depuis la derniere session:

Mémoires ef Trabaua, n" II-1951.

b) Sous presse:

Memoires et Travaux, $\mathrm{n}^{\circ} \mathrm{I}-1952$.

Annuaire Hydrologique de la France, 1950

Monographie hydrologique du bassin du Da Nhint, pat M. Slavitsix (Prix Henri-Milon 1951).

2 fascicules du Code d'essais relatifs à la mesure des débits par la méthode chimique et la méthode Grison d'une part, et par l'exploration du champ des vitesses, d'autre part.

c) En préparation:

Stations hydrométriques francaises $\left(3^{\circ}\right.$ partie : région des Pyrénées).

\section{Conférence du Comité Scientifique C.A.F.}

Il est porté à la connaissance des membres présents que M. D. Olivier-Martin, Ingénieur en chef des Ponts et Chaussées, Directeur de la Région d'équipement hydraulique Alpes II d'E.D.F., fera, vendredi 21 mars, à 21 heures, au siège du C.A.F., 7, rue La Boétic, une conférence sur les «Recherches récentes de glaciologie».

\section{COMMUNICATION DE M. SILBER}

\section{ÉTABLISSEMENT DE LA COURBE DE REMOUS D'UN BARRAGE EN COURS D'EAU NATUREL}

\section{Résumé}

Le mémoire a pour objet l'application à l'étude de la courbe de remous en cours d'eau naturel de la méthode générale d'étude et de tracés des lignes d'eau des écoulements permanents graduellement variès exposés à la Société Hydrotechnique. Celle-ci était fondée sur l'étude d'un cheminement dans un diagramme universel représentant, dans le plan de la profondeur réduite, $y^{*}$, en fonction du débit rédnit $q^{*}$ :

$$
\begin{gathered}
y^{*}=\frac{y}{\mathrm{H} s_{0}} ; q^{*}=\frac{\mathrm{Q} / l_{m}}{\mathrm{Hs}_{0} \sqrt{2 g \mathrm{Hs} s_{0}}} \\
\text { avec } l_{m}=\frac{S}{g}, \mathrm{Hs}_{0}=y_{0}+\frac{\mathrm{V}_{0}^{2}}{2 g}=y_{0}+\frac{Q^{2}}{2 g \mathrm{~S}_{0}^{2}}
\end{gathered}
$$

calculés en prenant pour unité de longueur une charge spécifique de référence arbitraire $\mathrm{Hs}_{0}$, les caractéristiques a charges spécifiques constantes dont l'une, celle pour laquelle la charge spécifique est $\mathrm{Hs}_{0}$, est la courbe universelle :

$$
q^{*}=y^{*} \sqrt{1-y^{*}}
$$

et les autres des courbes affines de cette dernière par une transformation analytique simple, ces caractéristiques ctant graduées en rapport $\mathrm{Hs} / \mathrm{Hs}_{0}$. 
Dans le cas particulier qui nous intéresse, deux difficultés nouvelles apparaissent:

$1^{\circ}$ Le radier du cour's d'eau est en général très tourmenté. La pente est très variable et imprécise. L'introduction de cette pente dans les calculs est une cause d'erreurs arbitraires.

$2^{\circ}$ La perte de charge en chaque section du cours d'eau est inconnue. Les données comprennent par contre les courbes hauteurs-débits en diverses stations, relevés sur des échelles pour différents débits soit des lignes d'eau. exprimés en cote N.G.F. avant l'établissement du barrage, la solution recherchée étant elle-mème la ligne d'eau après l'introduction du barrage exprimée en cotes N.G.F.

L'élimination de la pente du radier est obtenue en substituant l'équation :

$\mathrm{H}=z+\frac{\mathrm{V}^{2}}{2 g}=z+\frac{\mathrm{Q}^{2}}{2 g \mathrm{~S}^{2}} \dot{a} \mathrm{Hs}=y+\frac{\mathrm{V}^{2}}{2 g}=y+\frac{\mathrm{Q}^{2}}{2 g \mathrm{~S}^{2}}$

d'où l'on tire la même caractéristique universelle :

$$
\begin{aligned}
& q^{*}=z^{*} \sqrt{1-z^{*}} \\
& \text { avec } z^{*}=\frac{z}{\mathrm{H}}, q^{*}=\frac{\mathrm{Q} / l_{m}}{\mathrm{H} \sqrt{2 g \mathrm{H}}} \text { et } l_{m}=\frac{\mathrm{S}}{z}
\end{aligned}
$$

la cote z étant comptée à partir d'un plan horizontal bien choisi. Il en résulte l'utilisation du même diagramme universel représentant dans le plan de la cote réduite $z^{*}$ en fonction du débit réduit $q^{*}$ les mêmes caractéristiques graduées en rapport de la charge, soit de l'énergie, à une charge de référence arbitraire $\mathrm{H}_{0}$.

La méthode consiste alors à tracer dans ce diagramme le réseau des courbes représentant les lignes d'eau connues à débit constant avant l'introduction du barrage. La courbe d'utilisation après l'introduction du barrage, soit la nouvelle ligne d'eau, se déduit alors facilement de ce réseau en introduisant la seule hypothèse, classique et obligatoirement admise partout, que pour chaque section et pour une cote donnée de l'eau dans cette section le coefficient de CHezr est invariable quel que soit le débit. La méthode, extrêmement précise, ne comporte, à l'inverse des méthodes classiques, ni approximations successives, ni aucun tâtonnement.

Une application pour un exemple particulièrement compliqué est annexé au mémoire.

\section{Discussion}

M. le Président remercie M. Silber de sa communication.

M. Hupnen demande si la méthode diffère de celle qui résulte de l'application des équations classiques de Bous-
SINESQ. M. Hupner suggère, d'autre part, une vérification expérimentale de la méthode an cours d'une mise en chômage du bief amont d'un barrage.

M. Silber précise que la méthode qu'il a exposée est basée sur la théorie classique et sur les mêmes lois de variation des pertes de charge que les autres procédés de calcul, notamment celui des approximations successives; elle doit donc donner - et donne, en fait - théoriquement le même résultat, mais dans un temps plus ou moins long et avec une précision plus ou moins grande. La vérification peut être faite a priori, en comparant à la ligne d'eau effective d'un bief, la courbe de remous établie en fonction des données que l'on peut posséder sur l'état naturel de la rivière avant retenue; mais cette vérification présente une difficulté, c'est que l'on connaît mal, en général, la section en fonction de la profondeur d'eau.

M. VARLET demande combien de temps exige cette méthode, qui Iui paraît avoir sur les autres l'avantage de Ia précision.

M. Silber précise que son gros avantage est de mettre en cuvre un diagramme omnibus, sur lequel il suffit de porter les données. Le temps de mise en ordre des données et de leur représentation sur le diagramme est dífficile à chiffrer car il dépend de la complexité du problème posé. Les calculs eux-mèmes ont demandé ce travail préliminaire effectué dans le cas particulier annexé à la communication de 1 h. $1 / 2$ à $2 \mathrm{~h}$.

M. VARLET déclare alors que la méthode de M. Sriber est non seulement précise mais rapide.

M. le Président remarque qu'au point de vue visualisation, la méthode graphique est meilleure qu'un tableau de chiffres.

M. VArLet rappelle qu'une autre méthode graphique élégante qui traduit les équations successivement a été exposée dans la Houille Blanche, il y a quelque temps, a propos du barrage de Castelnau-Lassouts,

MM. Bourguignon et Sauvage de Saint-Marc donnent la référence de cet article : « Détermination graphique de la ligne d'eau et calcul des remous $\gg$, par N. RaYtchine et P. Chatelain, la Houille Blanche, mai-juin 1950, p. 573.

\section{COMMUNICATION DE M. SERRA}

\section{INTERPRÉTATION DES MESURES PLUVIOMÉTRIQUES - LOIS DE LA PLUVIOSITÉ}

Le mémoire in extenso de M. Serra et la discussion ayant suivi sa présentation en séance sont publiés pages 491 et suivantes du présent numéro.

La séance est levée à 12 heures.

\section{SÉĀNCE DU MARDI APRĖS-MIDI 18 MĀRS 1952}

La séance est ouverte à $14 \mathrm{~h} .15$ sous la présidence de M. Hupner.

\section{COMMUNICATION DE M. PEGGY}

\section{MORPHOMÉTRIE ET ÉCOULEMENT}

\section{Résumé}

La morphométric, etude statistique du relief: terrestre à l'échelle topographique sert, entre autres choses, à préciser l'interpolation qui intervient dans l'évaluation des termes de l'équation du bilan hydrologique.
En effet, ces deux termes, pluie et débit, sont habituellement connus ou mesurables, le premier $h$ à l'échelle locale, ponctuclle ou topographique (pluviomètres), le second $Q$ à l'échelle régionalee (stations hydrométriques propres à un bassin versant donné).

Une telle étude repose, en général, sur l'examen de la fréquence des altitudes (hypsométrie). On a proposé, plus rarement, de retenir la fréquence des pentes (clinométrie).

L'hypsométrie offre à l'hydrologie deux possibilités de recherches :

- d'un côté elle permet de pousser plus loin l'analyse des conditions d'écoulement sur un bassin en formulant et en verrifiant certaines hypothèses liant la hauteur 
d'eau écoulée à l'altitude $q=f(a)$ et permettant de déterminer les débits des cours d'eau situés dans les limites d'une région homogène d'après la fréquence des altitudes composant leur bassin versant Coutagne : Hydrométéorologic des bassins de haute montagne, pp. 17 et 18) : certains géographes retiennent souvent comme caractéristiques d'une région la pente de la courbe $q=f(a)$ que l'on nomme gradient d'écoulement.

- d'un autre côté, elle fournit par la comparaison globale entre les différents bassins des paramètres en fonction desquels on pourra étudier l'écoulement sur un groupe de bassins et aboutir à une loi $\mathrm{Q}=f(\mathrm{~A}), \mathrm{A}$ étant l'altitude moyenne des bassins.

L'auteur insiste sur cette deuxième ressource de l'hypsométrie et discute la fonction $Q=f(A)$, laquelle est perturbée par un certain nombre de « facteurs secondaires, plus on moins prépondérants », comme dirait M. Coutagne : proximité de la mer, limitation du déficit d'écoulement au-delà d'une certaine altitude, écart des diverses altitudes du bassin par rapport à la moyenne, elc.

Cette dernière considération conduit l'auteur à étudier les bassins versants en fonction, non plus de l'altitude moyenne, mais de l'encaissement moyen, défini comme la différence entre l'altitude moyenne du bassin et l'altitude moyenne dn talwog. L'auteur cite la Tinée dont le bassin présente entre le confluent du torrent de Saint-Dalmas et Isola une altitude moyenne de $1.920 \mathrm{~m}$ pour une altitude moyenne du talweg de $1.050 \mathrm{~m}$, soit un encaissement moyen de $870 \mathrm{~m}$ tandis que d'Isola au pont de Saint-Sauveur ces valeurs deviennent 1.800 et $705 \mathrm{~m}$ soit $1.100 \mathrm{~m}$ d'encaissement moyen. Comme on connait l'anomalie qui veut que l'écoulement s'amenuise ic d'amont en aval, les secteurs les plus riches en eau paraissent ètre ainsi ceux qui juxtaposent des vallées profondes à des sommets encore élevés.

La clinométrie est basée sur la « pente moyenne d'un versant $»$, défini comme le quotient de l'équidistance des isohypses par leur écart moyen, celui-ci étant le quotient de la surface comprise entre deux isohypses par leur demi-somme. Délicate et encore peu employéc, cette méthode ne doit être applicuée que dans le cadre de bassins assez peu étendus. Retenons seulement, parmi les résultats, les pentes moyennes ci-après :

Tinée, secteur cristallin .......... 0,61

Tinée, secteur sédimentaire . . . . . . 0,57

Guil supéricur $\ldots \ldots \ldots \ldots \ldots \ldots \ldots \ldots \ldots, 0,48$

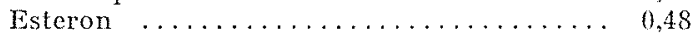

et remarquons que les deux derniers correspondent à des secteurs hydrologiquement pauvres.

La notion de pente moyenne intéresse aussi la pédologie et la biologie. On connaît le cas des montagnes méditerranéennes relativement bien arrosées, aui apparaissent néanmoins plus « arides » que les plaines paree que l'eau y ruisselle sans profit sur le sol. L'on pourrait songer à une formule d'aridité topographique combinant les pentes moyennes avec les données du climat.

\section{Discussion}

M. le Président remercie M. PÉguy et rappelle que sa communication s'apparente à celle présentée à la séance du matin par $M$. Senra; cette analogie permettra de reprendre la discussion de la communication de M. Serra interrompue faute de temps.

M. le Président demande à M. PéguY si la clinométrie intervenant dans son étude nécessite des cartes précises au point de vae altimétriques, celles-ci faisant actuellement défaut pour certaines régions.

M. PEGUY estime que les cartes utilisées doivent être précises et à l'échelle du $1 / 10.000^{\circ}$ ou $\mathrm{du} 1 / 20.000^{\circ} \mathrm{et}$, en attendant la sortie des nouvelles cartes préparées par I'Institut Geographique National, il suggère qu'un appareil liant un intégrateur et un stéréoantographe permettrait, s’il était réalisable, de calculer directement les volumes, les encaissements moyens et peut-être même les pentes des bassins sur les coupes des photographies aéricnnes stéréoscopiques.

M. le Président propose à M. PÉgux de se mettre en rapport à ce sujet avec l'I.G.N.

M. Senna signale qu'il a, lui aussi, remarqué la différence í contre-sens des débits spécificues de la Tinée a ux deux stations successives de Saint-Etienne à l'amont et de Bancairon à l'aval. Ayant calculé, par intégration des surfaces comprises entre les isohyètes, le volume d'eau tombé sur le bassin intermédiaire, il a expliqué l'anomalie ci-dessus par une différence des coefficients d'éconlement, due aux différences de nature géologique des terrains. L'encaissement maximum de la rivière dans cette partie de son cours, signalé par M. PÉGux, corres pond, selon M. Senra, à des terrains plus durs et plus imperméables, a travers lesquels la rivière a creusé son lit ì « coups de sabre $»$.

Sur la demande de M. Mreot, M. PÉguy rappelle la définition de l'encaissement moyen, et précise les données dont on dispose pour l'evaluer. Il signale qu'avant d'en venir à la notion d'encaissement moyen, qui est de Demangeon, il avait essaye, pour sa thèse, de prendre comme base une notion plus simple: l'altitude moyenne rolative, c'est-á-dire la différence entre l'altitude moyenne et l'altitude du point le plus bas de la section considérée. On peut prendre aussi l'écart quadratique moyen des altitudes, comme expression statistique de leur variabilité.

M. Mexot reprend l'observation de M. Serna qui Iui parât fondamentale : $\grave{a}$ encaissement moyen différent, il y a constitution géologique différente.

M. Pandé se demande dans quel cas le débit pent être le plus aftaibli : quand la rivière a un encaissement profond à gauche comme à droite, la partie qui est de faible altitude et qui est peu arrosée ne pèse pas beaucoup dans le calcul, et il doit en résulter un fort débit relatif; mais quand la partie considérée du bassin com. porte de vastes zones peu arrosées, du fait de leur altitude médiocre, c'est-à-dire lorsque l'encaissement au sens le plus mathématique du terme est réduit, le débit relatif doit être amoindri. L'intérêt de tout cela est de pouvoir évaluer les débits lorsqu'on ne dispose ni de jaugeages ni de moyennes exactes de pluie, directement observées sur un bassin et qu'on est réduit à préjuger de ces deux éléments d'après le seul zelief.

M. Chamayou rappelle que d'autres phénomènes interviennent dans l'écoulement: notamment la circulation souterraine émanant de bassins versants extérieurs, qui dépend de la géologie et peut atteindre $30 \%$ (rivières à bassin ealcaire du pays basque), et le « phénomène d'éponge », ou rétention, qui peut retarder de plusieurs mois l'écoulement (200 millions de $\mathrm{m}^{3}$ absorbés dans le bassin de la Dordogne inférieure, en aval de Marèges, et plus ou moins restitués après).

M. Senra précise que le phénomène d'éponge est très important peu de temps avant ou après l'averse, mais u'intervient guère dans les aclculs de moyenne portant sur une période telle que la nappe souterraine, constituée an début, soit équivalente à celle reconstituée en fin de période (équivalence des conditions anx limites).

M. Chamayou signale que, dans certains cas, le phénomène d'éponge joue sur plusieurs années : on a vu des sonrces taries à la fin de la période sèche et qui ont mis un peu plus d'un an à repréndre des débits à peu plès normaux.

M. le Président demande à M. Púcux si la loi de décroissance exponentielle du débit avec l'éloignement de la mer est sénérale, et s'il n'y a pas inversion dans certains pays à faible relief comme la Bretagne ou la 
Normandic oì l'on troure qu'au bord de la mer it pleut moins que 10 ou $12 \mathrm{~km}$ plus loin.

M. Pécier rappelle qu'il s'est réfélé à la loi exposée pall M. Serra au Congrès de l'U.G.G.I. à Bluxelles, el ajoute que pour une cote basse avec une plaine a l'arrière, il peut y avoir plusieurs maxima, 3 ou 4.

M. SenRa précise que la base de la variation n'est pas la distance à la mer mais la distance à une ligne parallèle à la cote pouvant être située à l'intérieur des terres; si bien que la courbe en cloche n'a pas son maximum rigoureux à la cote elle-meme, mais un peu plus loin : il y a d'abord croissance puis décroissance.

1. PÉGuY indique qu'en Bretagne les pluies sont assez mal connues parce que les pluviomètres, sénéralement placés sur les sémaphores, sont présumés ne pas donner une mesure conforme à la réalité.

M. Saxson confirme cet avis et, sur la demande de M. le Président, ajoute que la Météorologie Nationale n'a pas fait de nombreuses vérifications de la loi de variation de la pluie en fonction de l'écart à la mer; cette loi n'est pas vérifiée pour le littoral au nord de l'estuaire de la Loire et son hinterland.

M. Serra rétorque que le phénomène est cependant très net en Aquitaine d'après les cartes pluviométriques qui figurent sur les recueils donnés par la Météorologie.

M. Pardé confirme que la loi est vérifiée sur de nombreuses régions européennes et aux Etats-Unis où les moyennes isohyétiques et hydrométriques décroissent depuis les plaines côtières du golfe du Mexique à l'est du Mississipi (1.300 $\mathrm{mm}$ par an, module spécifique : $15 \mathrm{l} / \mathrm{s} / \mathrm{km}^{2}$ ) jusclu'au pied des Rocheuses (400 $\mathrm{mm}$, ouadis) avec débits moyens infimes. Mais dans le bassin de l'Amazone où malheureusement les pluviomètres manquent, e'est le contraire: à l'est on a des pluies de $1,30 \mathrm{~m}$ à $1,70 \mathrm{~m}$ et le gros corps du débit du fleuve semble provenir de la région moyenne qui, à $1.500 \mathrm{~km}$ de la mer, reçoit des masses de pluie plus grandes (2 à 3 mètres par an) que celles qui arrosent le bassin inférieur.

M. PÉGuY précise que les pluies des régions tropicales comme les bassins de l'Amazone et du Congo paraissent avoir des origines locales et que la loi d'écart ne vaut que pour les pays tempérés.

\section{COMMUNICATION DE M. B. GASPARD}

\section{NOTE SUR LA DÉTERMINATION DES DÉBITS DE LA SEINE A PARIS}

Le mémoire in extenso de M. Gaspard sera publié dans l'Annuaire Hydrologique de la France 1951.

\section{Résumé}

Les débits de la Seine étaient, avant sa canalisation qui remonte à 1865 , estimés en fonction de la hauteur. d'eau par des courbes comme celle de Porré au pont de lá Tournelle. Plus tard, en liant la pente et la hauteur d'eau, M. is Préauneau estimait assez exacte la formule $\Omega=110+180 \mathrm{H}+9 \mathrm{H}^{2}$, $Q$ étant le débit en $\mathrm{m}^{3} / \mathrm{s}$ et $\mathrm{H}$ la liauteur en mètres mesurés au-dessus du zéro de l'échelle du pont de la Tournelle.

Mais dès le milieu du $x^{e}$ siècle, le remous du barrage de Suresnes et la construction de ponts rapprochés dans la traversée de Paris ont pratiquement empêché d'appliquer les courbes ou formules antérieures, dans lesquelles II ne représentait plus qu'une fonction guère univoque de la pente et de la vitesse du fleuve. A chaque crue, sécheresse ou débâcle de glace, il fallait done procéder à un nouveau jaugease.

Malgré les difficultés inhérentes aux jaugeages, même grossiers (flotteurs lestés), sur un cour's d'eau large et frépuenté tel que la seine (danger pour les operateurs, interuption de la navigation, ete.), M. Lavi en 1928 el N. Gaspard en 1941 essayerent de reprendre l'établissement de coulbes analogues a celle de Ponsi, mais la précision resta précaire dans toute la zone des débits les plus courants. Cela tient, comme dit plus hat, à l'influence des ponts, qui est variable avec le débit mème, el a celle des barrages qui provoquent des débits très dilférents a l'aval, d'une part suivant le remplissage ou la vidange du bief amont et, d'autre part, pour une mème hauteur de chute, suivant la disposition des hausses ou des aiguilles. Enfin, les pompages importants effectucis en Seine à la traversée de la région parisienne et les pertes dans le lit majeur qui accompagnent les làchures des réservoirs à l'amont de Paris (restituées particllement et irrégulièrement suivant la saison) viement. encore troubler les mesures de debits malgre les soins apportés à levr exécution.

En présence de tous ces obstacles, et comme d'atutre part la senle solution rationnelle de jaugeages recls journaliers s'avère économiquement et pratiquement irréalisable, le service de la Navigation s'est rabattu sur l'évaluation du débit de la Seine tous les jours vers 9 heares da matin au barrage déversoir de Suresnes (passe rive droite), compte tenu des éclusées, de l'écoulement et des débordements par la passe navigable rive sauche. Grâce à des dispositions prescrites pour le barrage et accompagnées de jaugeages précis au moulinet suffisamment $\mathfrak{a}$ l'amont ou à l'aval, il a été possible d'établir des abaques comportant pour la mème disposition des hausses :

$1^{\circ}$ une échelle des hauteurs à l'amont;

2 une échelle des hauteurs à l'aval;

$3^{\circ}$ une échelle des débits correspondants.

Le Service de la Navigation a été ainsi en mesure do donner, depuis 1944, chaque matin le débit journalier de la Seine à Suresnes.

D'autre part, afin de vérifier l'importance des pompages et des restitutions dans le département de la Seine, le débit de la Seine à Suresnes a été confronté avec l'addition des débits de la Seine et de la Marne pris 24 heures auparavant à une distance telle de Paris que ces débits parviennent en mème temps à Suresnes, que l'on soit en période de crue ou de dérue. Il se trouve que les barrages de Meaux et de Vives-Eaux correspondent ì peu près à cette condition : pour éviter les frais de jaugeages coûteux suffisamment loin des barrages, ceux-ci ont été tarés sur modèle réduit ct permettent la comparaison avec Suresnes depuis le début de 1950. Compte tenu des débits du bassin versant intermédiaire (Grand-Morin, Essonnes, Orge et Yères), des pompages et des pertes, on a très souvent: débit Meaux + Vives-Eaux supéricur ì débit Suresnes + pompages aux alentours de Paris.

En serrant le probleme de plus près, il a été constaté que les abaques de Suresnes étaient moins précis (10 à $15 \%$ ) pour certaines zones de debits que les abaques établis pour Meaux et Vives-Eaux sur modèles réduits (5) à $7 \%$ ).

Cependant, la correction des débits de Suresnes par le total Meaux + Vives-Eaux n'était pas possible quotidiennement et M. Gasparp a été ainsi conduit à établir une fois pour toutes, en partant de ce double tarage, un barème domant les débits de la Seine, inférieurs à $1.100 \mathrm{~m} 3 / \mathrm{s}$ à la traversée de Paris, en fonction de :

$1^{\circ}$ La pente moyenne dans Paris entre le pont National et le pont d'Auteuil;

$2^{\circ}$ La cote du pont d'Austerlit

Ce barème a été établi en deux stades, comportant chacun plusieurs opérations.

Dans le tableat $I$, dont nous donnons un extrait cicontre :

a) On il classé les moyennes des débits journaliers 


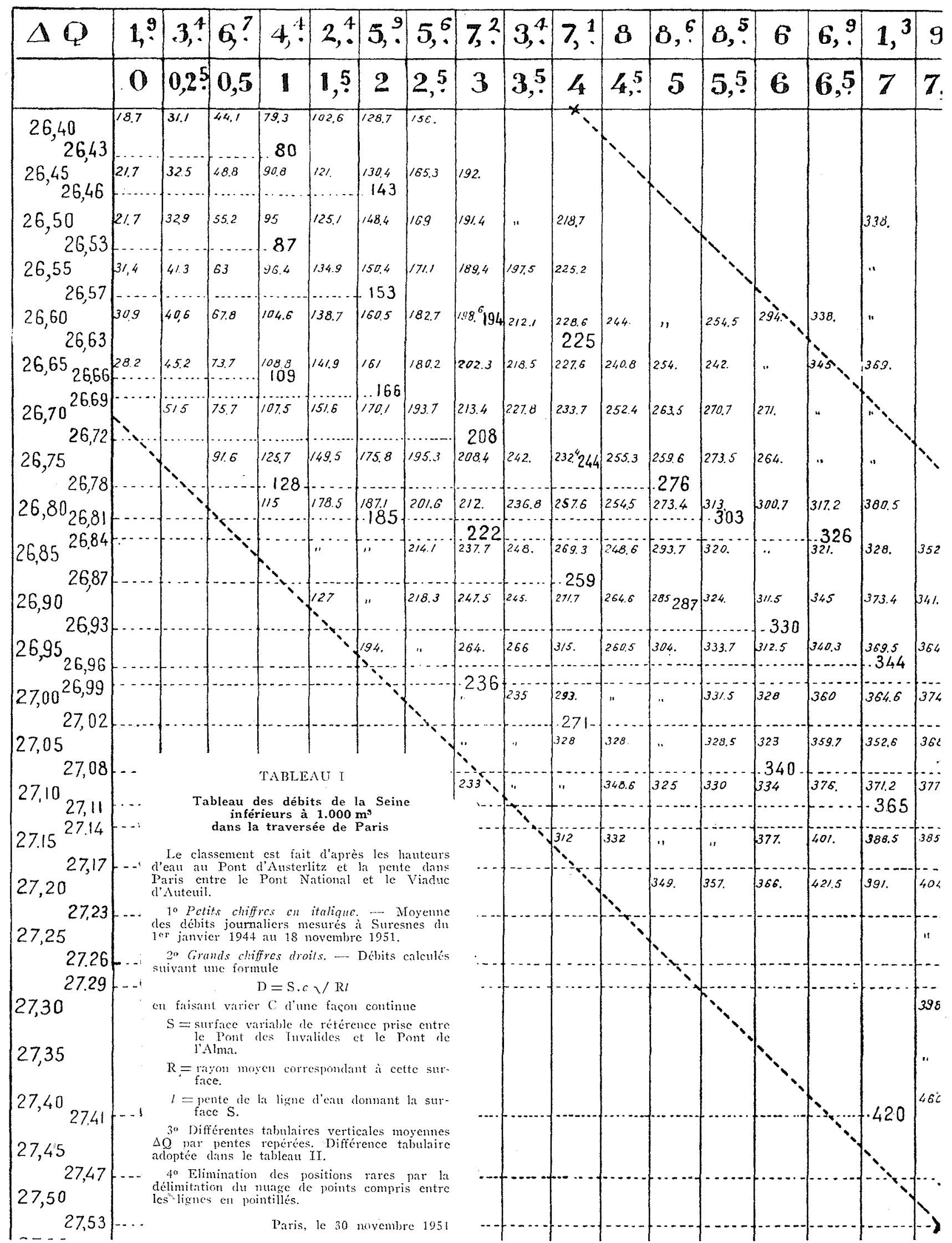


mesurés à Suresnes du $1^{\text {er }}$ janvier 1944 au 18 novembre 1951 dans des cases ayant pour abscisses les pentes de 5 en 5 millionièmes entre 0 et $16, \bar{\sigma}$, les cotes à Austerlitz de 5 en 5 cm entie $26,40 \mathrm{~m}$ et $29,20 \mathrm{~m}$.

b) On a calcule la moyenne des différences tabulaires verlicales, ou différences finies. $\Delta Q$ des débits correspondant à une meme pente et on a admis cette valeus moyenne comme constante pour cetle pente, en supposant la section du fleuve rectangulaire $(\Delta S$, done $\Delta Q$, proportionnels a $\Delta h$ ); ce qui conduit à la première ligne du tableau I.

c) En prenant comme section de référence me section du fleuve entre le pont des Invalides et le pont de l'Alma, on a calculé une variation fictive du coefficient $C$ de CHËzy à partir des éléments du tableau $I$ : débits connus de Suresnes, pente et hauteur d'eau $h$ (supposée égale at rayon moyen).

d) Entre les limites de cette variation, on a rectifié les valeurs de $C$ en s'imposant seulement que $C$ croisse avec le rayon moyen et la pente.

e) En repartant des coefficients $C$ ainsi déterminés, on a calculé, au moyen de la même formule de Chézy, des débits rectifiés destinés à donner des ordres de grandenrs, ou à servir de points de repère.

Dans le tableau $I I$, de mêmes coordonnées que le tableau I et dont nous donnons également un extrait ci-contre :

a) On a porté les moyennes de débits journaliers à Suresnes corrigées par leur comparaison avec l'addition des débits à Meaux et Vives-Eaux, par la considération cles variations des coles concomitantes a Joigny (Yonne), Damery et Chalifert (Marne) et compte tenu des points de repère du tableau $I$; ces rectifications ont permis d'éliminer certaines erreurs systématiques entachant ces débits.

b) On a ordonné les différences tabulaires horizontales ou différences finies $Q$ des débits correspondant à unc même hauteur d'eau et à des pentes variables en remarquant que ces différences décroissent comme croît la racine carrée de la pente (dérivation de la formule de Chízx par rapport à 1 ); on a ensuite interpolé ces dit-férences tabulaires au mieux.

c) On a corrigé les différences tabulaires verticales moyennes, en remarquant que ces différences croissent avec la pente comme la racine carrée de celte pente (dérivation de la formule de Chézy par rapport à $h$, la section étant supposéc rectangulaire et égale à $h_{1}$ et le rayon moyen égal à $h$ ).

d) On a considéré le tableau II comme un nuage de points et on $y$ a déterminé une fibre moyenne (dèbits soulignés ou pointés) : c'est sur cette fibre moyenne qu'on s'est efforcé de faire porter la croissance de la différence tabulaire verticale quand on passe d'une colonne à la suivante.

On a vérifié, au cours de la crue de décembre 1951janvier 1952, que le graphique des débits donnés par ce tableau prenait place entre le graphique des débits de Suresnes et celui des deux barrages d'amont, et cela en supprimant les pointes et creux incxplicables autrement qu'en les attribuant à des erreurs accidentelles.

Le tableau II, fruit d'une étude qui a duré plus de quinze ans, constitue done un barème établi en prenant comme appareil de mesure la Seine elle-mème et en utilisant, comme un jaugeur Venturi, son étranglement au droit des îles Saint-Louis et de la Cité. Si l'on considère qu'une étude sur modèle réduit de la traversée de Paris envisagée en 1941, aurait coûté 12 millions au cour's actuel, M. Gaspard a réalisé un double avantage : opération en vraie grandeur et économic.

Ce barème permettra de retronver les débits journaliers de la Seine depuis 1926 , date de son aménagement sous sa forme actuelle, et de les calculer dans l'avenir tant que de noureaux dragages importants n'auront pas été faits et que le bras de la Monnaie n'aura pas été ćlargi, comme il en est question.

\section{Discussion}

M. le Président remercie M. Gaspand de son importante communication et lui demande si les moyens actuels permettent de connaitre le débit d'une grande crue, comme celle de 1910.

M. Gaspard répond que la précision actuelle est de l'ordre de 5 it $7 \%$, tant pour les petits debits qui ont nécessité des corrections, faisant lobjet des tableaux présentés, aux abaques établies pour le barrage de Suresnes, que pour les débits supérieurs à $1.100 \mathrm{~m}^{3} / \mathrm{s}$ pour lesquels les courbes dérivées de celle de Porrú, et celles de M. LaNG étaient suffisamment précises. Pour les grands débits, on n'a plus un nuage de points, mais sensiblement une courbe, et l'un des points de celle-ci correspond à un jaugeage fait par le Service de la Navisation au moment de la crue de 1910. Le carré de la graduation de la conrbe établie en 1941 correspond a $100 \mathrm{~m} / \mathrm{s}$ (coordonnée horizontale) et a une hauteur de $20 \mathrm{~cm}$ (coordonnée verticale).

Sur la demande de M. Ginert, M. Gaspand précise que Ia crue de 1910 avait un débit de $2.350 \mathrm{~m} 3 / \mathrm{s}$.

M. Vennin demande si on a pu constater l'utilité des travaux exécutés dans le bassin de la Seine pour la régularisation de ce fleuve, notumment le barrage de Pannessière, sur l'Yonne.

M. Gaspand dit qu'il n'y a pas eu de grandes crues depuis 1950, date de mise en eau du barrage de Pannessière, mais que sá retenue, de l'ordre de 50 millions de $m^{3}$, ne paraît pas suffisante pour agrir efficacement contre les inondalions. Cependant, la lecture des courbes actuellement valables permet de se rendre compte, pour une crue donnée, de labaissement de niveau dû aux travaux de régularisation cffectués depuis 1910: on peut evaluer cet abaissement à $0,65 \mathrm{~m}$ environ, pour une crue de l'ordre de celle de 1910. Autrement dit, le coefficient $C$ de la formule de Crízy est légèrement supérieur, à l'heure actuelle, à ce qu'il était en 1910.

M. le Président estime que la question posée par N. Vennis pourrait faire l'objet d'une seconde communication, que M. Gaspard accepte de faire plus tard.

M. Senna demande si les débits journaliers de la Seinc à Suresnes, qui paraissent relevés règulièrement depuis 1944, d'après les tableaux présentés par M. Gaspand, pourraient maintenant être publiés dans l'Amnuaire Hydrologique de la France, comme débits de la Seine à Paris, avec rattachement des moyennes mensuelles à une grancie période et à la période de référence débutant en 1920, comme dans toutes les autres stations publiées dans cet annuaire.

M. Gaspard promet de remettre d'ici la fin de l'année les débits vérifiés après comparaison avee ses tableaux ct avec les informations des stations à l'amont, en vue de leur publication dans l'Annuaire 1951.

M. le Président Barrillon et M. Pardé se félicitent de cetle promesse qui permettra de donner à la fois plus de prestige à notre Annuaire Hydrologique et plus de continuité à l'étude des débits de la Seine.

M. Méo propose de rapprocher les résultats des jaugeages effectués par le Service de la Navigation (3e section) a la station de Bougival des chiffres obtenus at Suresnes par M. Gasparn, ce qui permettrait peut-être de préciser l'importance des prélévements par pompage qui sont restitués à l'aval, entre Suresnes et Bougival. M. Méo signale, d'autre part, que des échelles mal implantées ont pu faire croire qu'à l'aval de Paris la Seine coulait aussi à l'envers. 


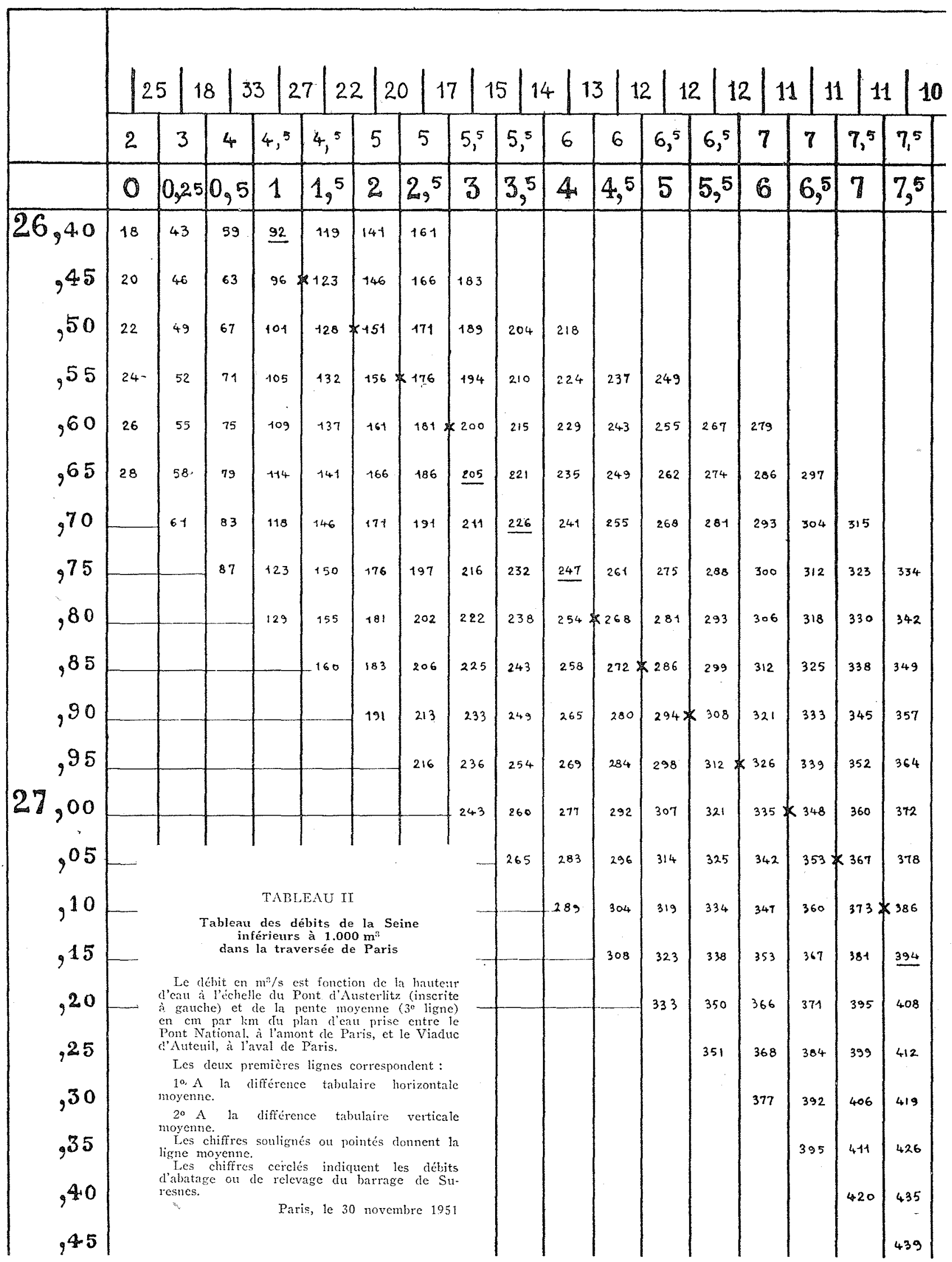


M. Gaspan précise qu'une telle erreur n'est pas à craindre pour la Seine dans la traverse de Paris car les échelles $y$ ont été vérifiées à $1 \mathrm{~cm}$ près, par référence. au N.G.F., sur Jes instructions de M. WATtrer, en 1934 et 1935 .

\section{COMMUNICATION DE M. DUFFAUT}

\section{CHOIX DU DÉBIT DE CRUE POUR LEQUEL IL CONVIENT DE PRÉVOIR LES OUVRAGES ÉVACUATEURS D'UN BARRAGE}

Le mémoire in extenso de M. Durfadr et la discussion ayant suivi sa présentation en séance sont insérés pages 506 et suivantes du présent numéro.

\section{COMMUNICATION DE M. PARDE}

\section{$1{ }^{\circ}$ LES DÉSASTRES DU KANSAS (1) ET DU MISSOURI EN MAI-JUIN 1903 ET JUILLET 1951 ET UN CATACLYSME} DE LA REPUBLICAN RIVER EN MAI-JUIN 1935

\section{Résumé}

Ces clesastres ont eu pour eause des pluies de saison chaude dues à des fronts contrastés entre des courants chauds provenant du golfe de Mexique et des masses d'air d'origine polaire. Ces averses, tombant sur des zones généralement peu arrosées, sont de deux types:

$1^{\circ}$ Le 31 mai 1935 un cloudburst atteignant des centaines de millimètres sur $2.000 \mathrm{~km}^{2}$ et 80 à $120 \mathrm{~mm}$ sur $10.000 \mathrm{~km}^{2}$ a provoqué sur la Kansas-River des maxima atteignant de 7 à $8.000 \mathrm{~m} \% / \mathrm{s}$ à Cambridge et Bloomington, mais s'aplatissant à 3 à $4.000 \mathrm{~m}^{3}$ à KansasCity.

Etendue du désastre : 100 morts, 20.000 têtes de hétail perdues, 500 ponts démolis ou endommagés, 1.485 maisons emportées.

2" En mai-juin 1903, apres une période de saturation, whe plaie décisive de 24 a 36 heures, mais non torrenticlle en général $(<133 \mathrm{~mm}$ ) tombant sur les moyens ct bas bassins de la Kansas-River et sur la basse SmookyHill a donné 6.000 à $6.500 \mathrm{~m}^{3} / \mathrm{s}$ au confluent de cetto rivière, 7.000 à $8.000 \mathrm{~m}$ à Manhattan, 9.000 à Topéka el 10.000 it Kansas-City.

Dégâts énormes, surtout le long de la Kansas-Rive: (100 morts, 16 ponts détruits à Kansas-City, 100 millions de dollars an cours actuel).

$3^{\circ}$ En juillet 1951, phénomène du mème genre qu'en juin 1903, mais plas fort : saturation plus efficace, débits énoumes et séries d'averses du 9 au 12 juillet, 3 paroxysmes séparés par des poses, surfaces frappées considérables (152 mm en 3 jours sur $130.000 \mathrm{~km} 2$ ).

Les zones les plus touchées sont á peu près les mêmes qu'en 1903, mais se situent un peu plus au sud-est, affectant la Neosho, affuent de l'Arkansas et l'Osage, dernier affluent notable du Missouri. Les maxima s'échelonnent entre $6.800 \mathrm{~m} 3 / \mathrm{s}$ sur la Smooky-Hill à Enterprise (cours inférieur) et 13.000 à $14.000 \mathrm{~m} 3 / \mathrm{s}$ à Topéka et Kansas-Cily.

Cette crue est digne de celle de la Garonne à Toulouse en 1875 , pour des surfaces comparables.

(1) Kansas-River : Affluent occidental du Missouri : BV $15.500 \mathrm{kn}$ i Kansas-City (confutent); dettx branches: Républican au nord $\left(6.500 \mathrm{~km}^{2}\right)$, Smooky Hill ait sud $\left(5.200 \mathrm{~km}^{2}\right)$ et 111 affluent $R G$., ha Bir-Blue $\left(2.500 \mathrm{~km}^{2}\right)$. Module assez faible $(155 \mathrm{~m} / \mathrm{s})$ et fort déficit. Alinentation pluviale assez médiocre $(400 \mathrm{~mm})$ dans les hautes plaines occidentales.
Les degàts atteignent 870 millions de dollars; il y a eu 110 morts (chiffe très réduit par la promptitude des secours et la bravoure des sauveteurs), un nombreux bétail péri, 17 grands ponts détruits, $2.256 \mathrm{~km} 2$ recouverts ( $=2$ fois la surface inondéc par le Pô en novembre 1951 ).

Un programme de protections locales et de réservoirs, partiellement réalisé, a sauvé cing fois la ville de Salina grâce au barrage de Kanapolis sur la SmookyHill; la réalisation totale aurait réduit les hauteurs maxima et évité le désastre des quartiers bas de KansasCity.

Des jaugeages extraordinaires ont été faits au cours de cette crue: à Topéka, $2 / 5^{\mathrm{e}}$ du débit passaient dans lo lit mineur et $3 / 5^{\circ}$ a travers le champ d'inondation; la vitesse sous le pont atteignait $2,20 \mathrm{~m}$ à la seconde. A Kansas-City, $4 / 5^{\circ}$ du débit passaient sous un pont et $1 / 5^{\mathrm{e}}$ sous un autre pont (décharge de la ville); la vitesse atteignait $5,8 \mathrm{~m}$ a la seconde. Pour la Neosho, qui a dépassé cinq fois les maxima connus, les $11 / 12^{e}$ du débit passaient par le champ d'inondation, et la vitesse dans le lit mineur atteignait $3,20 \mathrm{~m}$ à la seconde.

D'autre part, 50 ingénieurs appartenant au « Corps of Ingineers » de l'Armée et au Bureau of Reclamations ont travaillé a posteriori à étudier les circonstances exactes de la crue : ils ont supposé les averses tombant de $10 \mathrm{~km}$ en $10 \mathrm{~km}$ et calculé les débits qui auraient été provoqués par ces averses; ils ont procédé ainsi de proche en proche jusqu'à ce que la physionomic exacte du phénomène ait pu être reconstituéc.

\section{CRUE DE NOVEMBRE 1951 DANS LE E'ASSIN DU PO}

Le phénomène météorologique (commun aux crues conlemporaines du Rhone et du Pô est à deux temps:

$1^{\circ}$ Un afflux d'air méditerranéen chaud et humide, de direction $S$ et $S-E$, se heurte, ans la zone dépressionnaire du bassin du Pó, de la Provence et de la Ligurie, a un anticyclone d'Europe Centrale, avec isobares N-S el fort gradient barométrique E-O; il en résulte un ovale dépressionnaire à grand axe N-S ouvert côté nord.

$2^{\circ}$ Grâce à cet ovale, l'air chaud méditerrancen affronte l'air froid océanique venu de N-O et qui afflue it larriere de la dépression principale. Malgré son faible gradient barométrique, cette dépression secondaire déclanche, par suite de différences thermiques, des pluies diluviennes.

Parfois aussi, la dépression immédiatement coupable est un cyclone très creux, puissant et virulent.

En novembre 1951, les deux phénomènes s'associèrent du 6 an 11 et du 18 au 20 novembre: : cyclone très creux mais stable entre l'Espagne et les Iles Britanniques, atflux d'air humaide $S-E$ sur les Alpes françaises el le nord de l'Italie, mais déclenchement de la pluie seulement après formation de poches isobariques sur Ia Méditerranée occidentale, l'Espagne et l'Algérie sous l'influence des masses d'air froid arrirant de l'ouest.

\section{Pluies dans le bassin du Pô}

Tres grosses pluies périphériques contre les versants (nord-ouest et sud-ouest) et précipitations un pen plus intenses que d'habitude dans la plaine antour de Milan. la lame d'eau moyenne en 5 ou 6 jours sul l'ensemble du bassin padan l'emporte sur toutes ses devancières (216 et $236 \mathrm{~mm}$ ). La concentration habituelle des pluies se trouve placée vers l'aval, ce qui favorise la concordance entre les affluents.

D'autre part, le terrain est préalablement saturé par des pluies d'aout et d'octobre supérieures à la normale. La suite des averses rapprochées, soit par création de longues étales, soit grâce à des pointes successives, a rendu plus durables les gros débits des affluents. 


\section{Crues}

La plupart de ceux-ci, cependant, n'ont pas égalé leurs records (Tessin à Sesto Calende : $2.000 \mathrm{~m}: "$, Pò à Turin : $\left.1.050 \mathrm{~m}^{3}\right)$. Records battus : Trebbia : $1.630 \mathrm{~m}:$; Tanaro : 3.200 ; Sesia : 3.000 .

Le fleuve atteint $3.240 \mathrm{~m} 3$ a la sortic du piémont (Cascale Monferrato) et $8.790 \mathrm{~m} "$ à la Bocea (grossi de la Sesia, du Tanaro et de la Serivia). Ces chiffres avaient déja été dépassés, mais de peu, a la Bocea en mai 1926. La supériorité devient indiscutable à partir de Plaisance, apres le confluent de la Trebbia (Plaisance : $12.800 \mathrm{~m}$; Roncocorrente après te confluent de l'Oglio: $11.100 \mathrm{~m}^{3}$; Ponte Lagoscuro : 9.800 a $10.000 \mathrm{~m}$, y compris le déhit passant par les bièches).

\section{Ruptures de digues}

Plus de $5.000 \mathrm{~m}^{3}$ sont précicipités au nord, dans la riche eampagne de Polesine, après les ruptures qui eurent lieu à Paviole et Occhiobello dans Ia nuit du 14 au 15 novembre (inondation de $1.000 \mathrm{~km}^{2}$, ville de Rovigo menacée, ville d'Adria envahie, Adise rejointe, long éconlement jusqu'à la mer, à cause des remblais qu'il tallut faire sater; plus de 100 morts, plusieurs centaines do maisons écroulécs, nombreux bétail péri, fertile territoire ravagé).

Cependant les digues qui tinrent bon jusque vers Casalmaggriore (aval du confluent du Taro) contribuèrent i accroître les niveaux à l'amont jusqu'à la hautcur limite des digues en aval de Plaisance, surélevées parfois par des moyens de fortune. Les ruptures dautrefois. encore plus nombreuses, ont dirigé sur le champ d'inon-
Uation des debits plus puissants, mais on peul avancer avec certitude que les crues déjà mémorables de $190 \vec{t}$, 1917 et 1926 furent moins grandioses que celle de novembre 1951 dans l'ensemble.

Il faut remarquer que l'inondation de 1951 aurait été encore plus désastreuse sans l'influence atténuatrice des lacs subalpins (Lac Majeur, de Lugano, de Come, d'Iseo et de Garde) qui emmagasinèrent 1 milliard 200 millions de mètres cubes.

La vitesse de propagation de l'onde principale atteignit $5 \mathrm{~km} / \mathrm{h}$ entre la Bocea et Plaisance, apparemment $12,5 \mathrm{~km} / \mathrm{h}$ entre ee point et Crémone, of $5,7 \mathrm{~km} / \mathrm{h}$ entre Crémone et Ostiglia.

La monté maximum fat de 3 m en un jour a la Bocea. La grande hausse du 8 au 10 novembre fut suivic d'une baisse temporaire à la Bocea et d'un palier à Crémone, à l'aval. I)uléc de croissance totale : 5 jours et demi à la Boeca, 6 jours a Plaisance et Roncocorrente.

La seconde série d'averses, vers le 18 novembre, fut moins forte dans le bassin du Pó (sauf sur le Tessin el l'Adda) que dans celui du Rhonc. Elle prolongea, toutefois, la submersion.

La plupart des renseignements ci-dessus ont été empruntés à une étude très puissante du Service Fydrographique Italien parte en supplément du Bolletino Idrologico du mois de novembre 1951.

M. le Président exprime à M. Pardé ses remerciements pour son importante contribution à la session du Comite 'Technique, et les excuses de la S.H.F. pour le retard subi par son intéressante communication, par suite d'un décalage de l'horaire, et la nécessité consécutive de renoncet. ì la discussion, malgré tout l'intérêt qu'elle aurait pa présenter.

La séance est levée à $18 \mathrm{~h} .30$.

\section{SEANCE DU MERCREDI APRĖS-MIDI 19 MARS 1952}

Lat séance est omverte it 14 h. 30 sous la présidence de M. Ganuer.

\section{COMMUNICATION DE: M. TALORRE}

\section{L'ÉTAT ACTUEL DE LA TECHNIQUE DES CONDUITES FORCÉES SOUTERRAINES}

Le mémoire in extenso de M. Tarorre et la discussion ayant suivi sa présentation en séance sont publiés pages 513 of suivantes du présent numéro.

\section{COMMUNICATION DF M. CASACCI}

\section{ÉTUDE SUR LES FROTTEMENTS FLUIDES DANS LES TURBO-MACHINES}

\section{Résumé}

Le frottement des partics tournantes contre le fluide ambiant est souvent une source importante de pertes dans les turbo-machines; ainsi, les pertes par frottement fluide peuvent atteindre 3 a $4 \%$ ou plus de la puissance déreloppée par une Francis de petit nombre de tours spécifique. Cependant, l'étude des formes de « l'habillage $\Rightarrow$ de la roue et le calcul des dimensions des laby- rinthes permettent de réduire ees pertes dans d'assez grandes proportions pour les turbines Francis, les pompes centrifuges, etc.

La connaissance de ces pertes permet aussi d'eclairex certains points de la question si controversée : comment faut-il passer des caractéristiques du modèle à celles de la machine industrielle? Par exemple, pour les Francis doubles, les essais sur modèle réduit sont en généra! exécutés sur une roue simple. Il existe done sur le modele des pertes qui ne se reproduisent pas sur la roue industrielle. L'absence des pertes par frottement fluide et par fuite dans les labyrinthes coté plafond améliore le rendement de la turbine industrielle. Cette majoration de rendement peut atteindre 1 à $2 \%$ à pleine charge, soit 2 a $4 \%$ a demi-charge.

De mème, pour déterminer les caractéristiques de fonelionnement d'une Francis de haute chute, at l'aide de mesures sur modèle réduit, il est indispensable de faire une étude complète des pertes par frottement fluide et par fuite au joint labyrinthe pour la machine industrielle et pour la roue d'essais.

Pour la présente étude, nous préciserons d'abord les juissances absorbées par des corps de révolution de différentes formes tournant dans un milien fluide limité par des parois de formes et dimensions diverses. Nous analyserous de même quelle et l'influence de la rugosité des parois mobile ou fixe sur la puissance absorbece. Nous appliquerons ensuite ces résultats d'essais aux calculs de la perte par freinage des parties tourmantes it un cas concret. 
T. - Puissance absorbée par un cylindre tournant dans un milieu fluide infini ou limité par une paroi cylindrique cocriale fixe.

1-1. - Cas du cylindre loumant dans an milieu fuide infini :

Soicnt $\tau$ la contrainte de frottement, $V$ la vitesse tangentielle du cylindle, $R$ le rayon du cylindre; on peut definir le coefficient de froltement par:

$$
C_{j}=\frac{\tau}{1 / 2 Q V^{2}}
$$

Des essais exécutes par plusienrs expérimentateurs permettent de détermincr $C_{;}$en fonction du nombre de liErouds. $R=\frac{V R}{v}$ et pour differentes rugosites superficielles du cylindre. Applications: puissance absorbec par les arbes des francis doubles, etc.

\section{[-2. - Cas des cylindres coaxianx.}

Soit $h$ le jeu radical entre les deux cylindes, $R_{1} R_{2}$ les rayons des cylindres intericur et extéricur.

$$
c_{f}=\frac{\tau}{1 / 2 Q V^{2}} \text { est fonction du nombre de Reynoms }
$$
$\Omega_{1}=\frac{V R_{1}}{v}$, de l'entrefer relatif $h / R_{1}$ et de la rugosité superficielle des cylindres.

Pour des $h / R_{1}$ supérieurs à $1 / 1.000$, le cas du cylindre intérieur tournant, cylindre extérieur fixc est essentiellement différent du cas cylindre intérieur fixe cylindre extérieur tournant.

\section{II. - Puissance absorbée par un disque tournant dans un milieu fluide infini ou limité par un carter enve- loppant le disque.}

II-1. - Disque tournant dans un milien fluide infini.

Soit M le couple tendant à s'opposer à la rotation du disque, $R$ le rayon du disque, $\omega$ la vilesse de rotation; on peut définir le coefficient de frottement par :

$$
\mathrm{C}_{f}=\frac{\mathrm{M}}{1 / 2 \mathrm{Q}^{5} \omega^{2}}
$$

Le calcul permet de déterminer $C_{i}$ en fonction du nombre de Reynolds $\mathcal{Q}=\frac{\mathrm{VR}}{v}$; on obtient $C_{j}=\frac{3,68}{\sqrt{R_{L}}}$ pour $R$ inférieur à 310.000 environ; au-delà $C_{f}=0,146$ R-1/s (Karman, Cochran). Les essais de plusieul's expérimentateurs confirment ces résultats.

\section{II-2. -- Disque tomnant dans un milien fluide limité par une enveloppe.}

Soit s la distance entre la paroi da disque et de l'enveloppe, $\mathrm{C}$, depend de $\mathcal{R}$ et de $s / R$. Pour les grands nombres de Reynotos, $C_{f}$ est minimum pour $s / R$ voisin de 0,005 .

$\mathrm{C}_{f}$ est, bien entendu, fonction de la rugosite des parois du disque et de l'enveloppe. Pour un disque lisse tournant dans une enveloppe rugueuse, l'augmentation du coefficient de frottement est environ égale au $1 / 4 \mathrm{de}$ celle que l'on constate dans le cas d'un disque rugueux tournant dans une enveloppe 1'uguense. Si l'on peut quelquefois laisser les parois fixes brutes de fonderie, il est indispensable d'usiner les parties toumantes.
Application. - Puissance absorbée par les flasques, la ceinture, le plafond de la roue, ete.

Conclusion. - M. Fontane a réalisé toute une serie d'essais sur la séparation des pertes dans une turbine Francis. Il a en particulier déterminé les pertes pal eflet de freinage des parties tournantes a l'aide de la mellrode thermométrique. Ce sont, à notre connaissance, les seules mesures de ec genre qui aient élé efrectuées sur un turbine industrielle. La machine ayant fait l'objet des essais développe 42.500 ch sous $255 \mathrm{~m}$ de chute en toumant à 500 tours $/ m$.

11 nous semble intéressant de comparer les pertes mesurées par M. Fontane avec celles que l'on peut calculer à l'aide des essais réalisés par différents expérimentatemes.

Pour caculer ces pertes, nous assimilerons le plafond ct la ceinture de la roue à des disques lisses de $1.940 \mathrm{~mm}$ de diametre. Pour une distance moyenne entre paroi fixe et mobile de $70 \mathrm{~mm}$ et pour un nombre de Reynoros $\left(R=4,3.107 . C_{f}\right.$ est voisin de $2,5,10^{-3}$. Lál puissance absorbée par le plafond et la ceinture atteint done:

$$
\mathrm{N}=\mathrm{C}_{\mathrm{f}} .1 / 2 \mathrm{QR} \mathrm{R}_{1}{ }^{5} \omega^{3}=212 \mathrm{ch}
$$

La puissance absolbec par frottement fluide daus les labylinthes s'exprime de mème par :

$$
\mathrm{N}=\mathrm{C}_{f} 1 / 20 \mathrm{SV} s
$$

$S$ étant la surface latérale des labyrinthes. Ceux-ci sont constitués par deux cylindres de $205 \mathrm{~mm}$ de hateur et $1.940 \mathrm{~mm}$ de diamètre. Pour un jeu radial de $1 \mathrm{~mm}$ et pour un $\mathcal{R}=4,3.10 \pi, C_{\text {, }}$ est voisin de $1,6.10^{-3}$. La puissance absorbée dans les labyrinthes atteint donc $\mathrm{N}=360 \mathrm{ch}$.

Enfin, la puissance absorbée dans le labyrinthe situe à la sortie de la roue et du côté fond, s'élève à :

$$
\mathrm{N}=\mathrm{C}_{i} 1 / 2 \mathrm{QSVB}=35 \mathrm{ch}
$$

avec :

$$
\begin{aligned}
& \text { diametre du labyrinthe : } 1,5 \mathrm{~m} \\
& \text { hauteur }: 0,105 \mathrm{~m} \\
& \text { jeu radial environ : } 2 \mathrm{~mm} \\
& \mathrm{C}_{f}=1,7 \quad 10^{-3}
\end{aligned}
$$

La puissance totale absorbée pal frottement fluide s'élève donc à : $212+360+35=607 \mathrm{ch}$.

Les pertes mesurées par M. Fontane, lors d'essais réalisés sur une roue mumic de labyrinthes finement polis, atteignaient $650 \mathrm{ch}$, soit $1,53 \%$ de la puissance de la machine.

Les mesures effectuées semblent donc confirmer de façon satisfaisante les calculs. Nous nous proposons d'ailleurs de procéder à toute une campagne de mesures sur des Francis de haute chute, munies de roues ayant un «habillage » et des labyrinthes de formes simples. Ces mesures nous permettront de comparer avec exactitude les pertes par frottement fluide pour le modèle réduit et pour la turbine industrielle.

\section{Discussion}

M. le Président remercie M. Casacca d'avoir fait uno bibliographic considéable des pertes par frottement fuide dans une turbine. Le rapprochement qu'il a présenté des résultats d'essais innombrables éclaire la question si controversée de savoir comment il faut passer du petit au grand. L'exposé de M. CAsaccr prouve notamment que les essais sur modèle d'aubages seuls ne suffisent pas et que ceux-ci doivent ètre associés à des essais de modèles de rones et même de turbines complètes.

M. Tenot donne les résultats d'experiences qu'il effec- 
lue depuis deux ans pour montrer la complexile du probleme de la turbulence daus ses rapports avec la viscosité; l'influence de l'échelle de la tutburlence que M. le Président Ganer lui avait signalée alors qu'il étail jeune ingénicur aux Ateliers Neyret-Beylier et PiceardPiectet à Grenoble, a été évoqué récemment par le docteur WILLE à un récent Congrès de Mécanique des Fluides, tenu à Berlin en férier 1952, et soulevé, dans un tout antre domaine, par M. le Professeur Dognon, de la Faculté de Médecine de Paris, à propos de la puissance nécessaire à la production des ultra-sons dans les liquides, puissance qui serait 100 fois supérieure à celle qui est donnée par la formule elassique de Newton.

M. TENOT signale notamment des anomalies constatées dans ses experiences persomelles sur des disques tournant a faible vitesse relative dans un milieu fluide limité par des parois fixes très rapprochées du disque tournant (fig. 1). Ios nombres de Reynomos caractéristiques étant

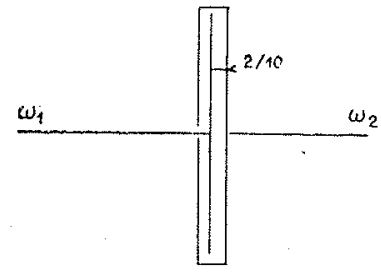

Fik. 1.

inférienr's à 50, la loi de Newron est rigoureusement satisfaite pour des disques rigides pleins, mais non avec des disques minces percés de trous on munis de fentes déterminant un voilement (fig. 2), lesqucls obẻissent ì la loi quadratique.

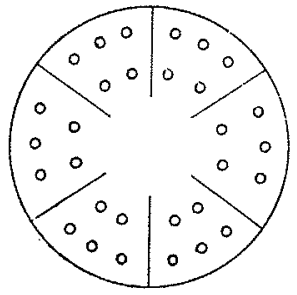

Frg. 2.

M. 'Teno' rappelle à l'appui de ses résultats certaines remarques que lui avait faites M. KanPE DE FEHET, il y a environ vingt-cinc ans concernant l'interprétation de la validité des équations de Navien-STokes. Si MM. Fonter et Comoler ont montré, aux premières «Journées de l'Hydraulique $\gg$ (juin 1949), que le nombre de Rrynolos criticue peut être prolongé jusqu'à 120.000 , il faut noter que cela n'est possible qu'au prix de très grandes précautions expérimentales. Les lois de Newron sur la viscosité ne sont vraies qu'à la limite, où $v=0$; en fait, il y a toujours dans la loi réelle des termes complémentaires plus au moins importants suivant l'agitation et il y aurait lieu de définir ces facteurs complémentaires et leur échelle par rapport à la viscosité fondamentale.

Sur deux demandes de M. Hupner, M. TEnot précise que les épaisseurs minima de fluide sont de $2 / 10^{\circ}$ à $1 \mathrm{~mm}$ mais déclare d'antre part que ces expériences ne lui paraissent pas pouvoir apporter quelques lumières sur le grippage des pivots des alternateurs.

M. le Président pense que les divergences constatées par $M$. TENoT pouraient provenir d'une détermination imprécise de la dimension linéaire intervenant dans le nombre de Reynoms dans le cas du disque peré de trous.
M. Casacici estime que les essalis de M. Tenot ne sont pas tout a fait comparables à ceux qu'il a exposés cal pour ces derniers la distance du disque mobile à la paroi fixe atteignait plusieurs millimètres tandis qu'elle n'était que de $2 / 10^{\circ}$ de millimètre dans les expériences de M. 'Texot.

Lorsque la distance entre la paroi et le disque mobile est relativement importante (quelques millinètres) les formules établies théoriquement pour le régime laminaire sont confirmées par les essais jusqu'a des nombres de linyoldos voisins de 104 , le nomble de Reyolds étant défini par la vitesse tangentielle du disque, le rayon du disque et la viscosité cinématique du thuide. Au-dela de ces nombres de Rexnoros le mécanisme de l'éconlement so compliciue. Les mesures effectuées par Schulz-Grunow montrent qu'il se forme au voisinage du disque mobile el de l'enveloppe fixe une couche limite où les gradients de vitesse sont élevés. Le fluide est chassé vers la périphérie dans la couche limite contiguë an disque, tandis qu'il s'ecoule vers le centre dans la couche limite au voisinage de l'enveloppe. L'annea fluide compris entre tes deux couches limites toune en bloc à une vitesse angulaire inférieure a celle du disque.

M. Casacci fait remarquer que dans le cas de l'écoulement d'un flude entre deux cylindres coaxiaux en mouvement relatif et lorsque seul le cylindre intérieur tourne, on constate que le couple mesuré est supérieur a celui déterminé par le calcul a partir de nombres de Reynolds tres faibles. Le nombre de Reynolds est ici défini par la vitesse tangentielle du disque, le jeu radial et la viscosité cinématique du fluide. Le nombre de Reynoms critique peut être calculé par le critère de Taylor qui a été vérifié expérimentalement de façon très satisfaisante.

M. Casacci pense cependant que les divergences constatées par M. Tenor sont probablement imputables à des imperfections d'usinage du disque.

M. Tenot précise que la même constatation a été faite cn régime établi ct après suppression du voilage.

M. Fontare indique qu'il a eu l'occasion de mesurer cxpérimentalement les pertes faisant l'objet de la communication de M. Casacci et croit savoir que les résultats de ces mesures correspondent à peu près exactement au calcul de M. Casacci.

M. Fontane précise que ces essais ont porté sur des entrefers qui descendaient jusqu'à environ $8 / 10^{\circ}$ de $\mathrm{mm}$ (turbines de Brommat).

M. le Président Garmex pense qu'il serait intéressant de ponrsuivre les expériences dans lesquelles M. FONTANe a réussi à séparer les pertes, et de confronter les résultats avec ceux que M. GASAcci a présentés.

M. Fontane accepte de présenter ses propres résultals laccord avec M. Casaccr.

M. Tenot propose quant à lui, d'exposer en détail, dans une prochaine séance, quelques-uns des résultats dont il vient de donner un apergu d'ensemble (coupleur à viscosité, ayant fait l'objet de la discussion ci-dessus, infuence de la turbulence sur le nombre de Rrysorns eritique iuférieur, effet Weisserneng démontrant la combinaison des lois de Newron et de Hoore et conduisant ¿ Ja loi de Maxwhict) (1).

M. Desmur signale que la formule de Moony a parfois donné lieu à des mécomptes (articles des A.S.C.E. et A.S.M.E. Transactions 1941) et le « Hydraulic Institule» devait publiel de nonvelles règles avec une discussion de cette formule.

Si l'application de la formule de Moony est relativement facile pour les turbines, pour lesquelles la hauteur theorique se déduit aisément de la puissance sur l'arbre,

(1) Comme il a ité conventu d'entendre M. Fontainc et M. Tenot en détail sur ces questions, nous nous bornons à reproduire linterven. tion de $\mathrm{M}$. Tenct sur la première, les deux autres n'ayant d'ailleurs pas été suivies de discusion. 
clle est inextricable pour les pompes où la hauteur théorique doit être déduite de la puissance absorbéc diminué des pertes mécaniques. Cette formule admet a peu près le mème coeffieient de rugosité que la formule de Blasivs.

M. Casacci pense qu'il n'est pas possible de trouver une formule universelle qui permette de calculer les majorations de rendement pour les turbines et pour les pompes.

M. Texor estime cependant gue fe rendement hydratlique devrait suive a peu près la formule de Moovy.

M. Casacci signale que pour les turbines Francis de pelits $N_{s}$ la majoration de rendement imputable à la roue seule est probablement négligeable si le modèle de dimensions convenables a été soigneusement usiné. Les rendements de la roue d'essais et de la roue industrielle peuvent atteindre 97 à $98 \%$.

M. Fontane signale que dans les turbines de faible $\mathrm{N}_{,}$, on a remarqué qu'avec des aubes usées par cavitation, et mème trouées, il n'y avait pas de baisse de rendement de la roue.

M. Casacir fait remarquer qu'il est difficile de définir l'efficience de l'écoulement dans les aubes des turbines Francis de petits $N_{3}$. L'énergie cinétique du mouvement relatif est très faible par rapport à la puissance totale transformée. Les pertes dans les aubages, exprimées en fonction de l'énergie einétique du mouvement relatif, ne peuvent donc ètre très importantes. C'est ce que semblent prouver les mesures effectuées par M. Fontaine sur une lurbine de $\mathrm{N}_{s} 110$ a $80 \%$ de la charge. Pour cette machine l'énergie cinétique du mouvement relatif représente quelques millièmes de l'éner'gie totale transformée.

M. le Président sonligne les difficultés de ces transpositions et l'intérêt de la communication de M. CASACCr qui ouvre la porte sur certaines clartés relatives à ce sujet.

\section{COMMUNICATION DE M. FERRANDON SUR LE MÉCANISME DES TERRAINS PERMÉABLES}

\section{I. -- Econlement de l'eau dans les terrains perméables}

M. Barrillow rappelle que l'exposé de M. Fermañon ne constitue que la première partie d'un ensemble important et susceptible d'intéresser le Comité Technique. Cet ensemble devant être publié par ailleurs, nous reproduisons simplement la discussion qui a suivi l'exposé.

M. Fenraydon ajoute deux des nombreuses applications de la théorie dérivée des conditions d'intégrabilité de Cauchy qu'il vient d'exposer: la détermination de la profondeur limite de dragage d'un bassin et le tracé des courbes d'équilibre et d'égale résistance au glissement d'un talus, notamment dans le cas d'un talus alimenté.

\section{Discussion}

M. le président remercie M. Fenrandon des nombreux resultats pratiques qu'il vient de présenter.

M. Hupven fait remarquer que cette theoric suppose que l'écoulement obéit à la loi de DARcy qui est notamment satisfaite pour les régimes laminaires (c'est-à-dire de Poiseúlale); n'y a-t-il pas des cas où l'écoulement dans les terrains véritables obéit à la loi de Nounter (puissance $1 / 2$ de la charge)?

M. Fernandon indique qu'en effet lorsque le gradient $\$$ est suffisamment grand la loi laminaire n'est plus valable et lat théorie tombe. Dans les terrains pulvérulents, cela arrive pour les grosses différences de charge, mals dans les argiles ou les marnes où te coefficient de perméabilité est faible, la loi de Poiseunle reste valable même avec de forts gradients. Une étude critique sur la validité de l'hypothèse de la loi de Darcy se trouve, d'ailleurs, dans l'exposé général de M. Frrmandon.

M. le Président signale qu'il y aurait intérèt à confronter ces résultats thériques avec ceux de séries d'expériences.

M. Fentandon est bien de cet avis et souligne d'ailleurs qu'en toute rigueur l'expression du gradient devrait comporter un terme $\mathrm{U}^{2} / 2 g$ faisant intervenir l'énergie cinétique: or, ce terme a été négligé dans l'exposé. devant la charge statique.

M. Ham pense, qu'en fait, la question ne se pose pas dans le cas des grandes vitesses de circulation, celle-ci dtant acompagné le plus souvent par les phénomènes de boulance, c'est-a-dire de destruction du milieu sous l'effet de gradient relativement faible. Pratiquement, tant que ce stade n'est pas atteint, l'écoulement est bien régi par la loi de Darcy. C'est plutôt du côté des très faibles vitesses de circulation dans les milieux très peu perméables que l'hypothèse de Darcy est critiquable; des essais suédois semblent avoir montré l'existence d'un scuil de vitesse se traduisant par un gradient limite au-dessous duquel l'éconlement n'aurait pas lieu. De même, M. Buisson a indiqué, notamment pour la vase do Tunis, un effet de blocage capillaire de l'eau intersticielle liée aux particules d'argile. D'après M. Duniez, Ijirecteur du Laboratoire des Ponts et Chaussées, ce blocage serait fonction de linverse du rayon de courbure des tubes capillaires.

M. Ferrandon précise qu'il a indiqué les deux seuils de validité dans son étude critique. Il trouve d'ailleurs très judicieuse l'intervention de M. HaBn, qu'il interprète comme suit :

On peut admettre que le massif est formé d'un certain nombre de grains entourés d'eau fixéc à ceux-ci. Le coefficient de perméabilité est alors fonction de la viscosité du liquide libre et de la structure géométrique du milieu où ce liquide filtre; or, cette structure est variable puisque, en plus de l'édifice solide, elle comporte de l'eau fixée en fonction du gradient qu'on applique au dispositif, e'est-à-dire des conditions d'alimentation et de restitution.

En conclusion, M. Ferranoon indique que le phénomène est complexe et que le schéma qu'il en a donné pent être, au-delà des seuils évoqués ci-dessus, assez éloigné de la réalité. Entre ces frontières, celle-ci est convenablement représentéc, ef en tout état de cause utilement esquissée l'allure générale de l'écoulement et des actions qu'il détermine dans le massif où il intervient.

La seance est levee à 18 h. 30 . 


\section{Séance d'information générale du 21 mars 1952}

\section{General Information Meeting}

La Société Hydrotechnique de France a organisé, lo 21 mars 1952, salle Marcean-Chaillot a Paris, sur l'initiative et sous la présidence de Mi. Maurice Garres, uno séance d'information générale en vue de faire ressortir les services que ses travaux penvent rendre dans quel(tues-uns des multiples domaines où la science de l'Hydraulique trouve son application.

A la tribune, aux côtés de MM. Barrillox, Président d'honneur et Président du Comité Technique, et Garmer, Président de la S.H.F., avaient pris place MM. AmLenet, Vice-Président de la S.H.F., Hupser, DE Rouvilla, Nizery et Messines du Sourbier, Membres du Conseil d'administation et Présidents de Sections de la Société.

Parmi les persomalités qui avaient répondu à l'appel de notre Association, soit en assistant à la séance, soit en s'y faisant représenter, nous avons pa noter :

Mme Michel Gavper, fille de notre regretté Président, M. Henri Mmox.

MM. Caquot el Darrieus, Membres de l'Institut.

MM. Aubert, Blanc, Le Bourhis, Chevrier, Coyne, Marx, Masse, Mrae, Membres du Conseil d'administration de la S.H.F.

M. Fenneworin, Secrétaire général; M. Rabrcuer, Président de Commissions et M. Reminueras, Secretaire général du Comité technique de la S.F.F.

M. Ch. Beat, Inspecteur genéral des Ponts et Chaussées. M. Suduet, Inspecteur général des Ponts et Chaussées.

M. Varlet, Inspecteur général des Ponts ef Chaussées.

M. Bollaert, Président de la Cie Nationale du Rhône.

MM. ne Maublanc. Contrôleur général, et Libarrs, exDirecteur regional d'Equipement à E.D.F.

M. Namy, Chef du Service de Ia Construction du Matériel it F.D.F.

M. ANorve, Inspecteur général pour l'Union Françase à E.D.F.

M. Tarome, représentant M. Roussebjer, Chef du Service «Projets hydro-électriques » à E.D.F.

M. Senns, Chef de la Division «Hydrologie » du Service des Etudes et Recherches Hydrauliques d'E.D.F.

MM. Dacarrier, Dhames, Gourdier et SaUvage de SaintManc des Els Neyrpic.

MM. P. Huverix, 'Thimed et Ch. Rabaun de la Sté Generale d'Entreprise.

MM. François Ossude et Vaillant de la Sté Ossude.

M. Benoist des Chaudronneries des Pyrénées.

M. Paul Bergeron de la Maison L. Bergeron.

I. de SaINT-Vaunry de la Cie Sulzer.

M. Chamayov de la Société Hydro-Electrique du Midi.

M. Dumanots de l'O.N.E.R.A.

M. Lagros du Burean d'Etudes Coyne et Bellier.

M. Ponsar de la Sté Hydraulique et Urbanisme.
M. Duffaut, Chef du Service technique de l'Energie Electrique et des Grands Barrages, M. Lamouroux, Chef de la $I^{\text {re }}$ Cireonscription électrique et M. Mirot, Ingénieur au Service des Grands Barrages.

M. Gment, Directeur du port de Nantes.

M. Hurauir, Inspecteur général gẻographe.

M. Koch, Directeur technique des Eaux et de l'Assainissement à la Préfecture de la Seine.

M. Larriev, Inspecteur géneral des Eaux et lorèts.

M. Cherney, Conservateur des Eaux et Foróls.

M. Gravevx, Inspecteur général du Génie Rural.

M. le Général Jovver, Commandant l'Ecole Polytechnique.

M. Rovcher, Président Directeur genéral des Ateliers et Chantiers de Bretagne, représenté par M. Prver.

M. Baffoun, leprésentant M. Pronger, Directeur de la Sté d'Entreprises générales et de Travaux publics.

M. Bordes, de la Sté Dumez.

M. Parisor, Directeux général du « Soliditit Français ».

M. Cl. Rabavd, de la Sté de Construction des Batignolles. MM. Lersive, Président Directeur général et R. Secraud de la Sté Desplats et Lefèvre.

M. Chabal, Président de la Sté Chabal et Cie.

M. Francois Garch, de la Sté des Produits Azotés.

M. Guinet, de la Cie Pechiney.

M. Companon, Directeur des Etudes, et Manie, Professeur à l'beole Centrale des Arts et Manufactures. ete., etc.

Quelques autres personnalites avaient tenu d s'excuser de ne pouroil assister ¿t la séance, notamment:

ins.

Camcher, Président honoraire du Comite lechnique de la S.H.F.;

Avgustri-Nomman, Président des Chanticrs et Ateliers Augustin-Normand.

Bigorcine, Président du Bureau central d'Etudes pou' les Equipements d'Outre-Mer;

CaDEr, Directeur général des Chabonnages de France.

Carver, Inspecteur général des Eaux et du Génie Rural.

Delatrre, Directeur général de la Cie Nationale du Rhône.

Devun, Directeur des Services fnanciers d'E.D.F.

Donges, Secrétaire génélal aux Travaux publics.

Escrancion, Directeur de l'Institut Polylechnique de Grenoble.

Ferrand, Directeur de la Sté Dauphinoise d'letudes $\cdot($ de Montages.

Gaden, Directeur de l'Atelier des Charmilles à Genéve.

Gexrmar, Président de la Sté des Transports pétroliers par pipe-line.

Gougranken, Ingénieur hydrographe en chef. 
Grapr, Directeur du port de Strasbourg.

Graveux, Inspecteur général des Eaux et du Génie Rurat. Gravier, Directeur général de l'Energie Electrique du Maroc.

J. GrolÉE, Directeur adjoint de la Cie « Pechiney ». Inage, Président de l'Union Technique de l'Electricité. Lucas, Directeur de l'Eeole nationale du Génic Rural. Matcon, Président de la Sté Francaise de Métallurgie.

Après une allocution brillante et substantielle à la fois de M. le président Garres sur « la multiplicité des domaines intéressés par l'hydraulique », MM. Nizeny, Hupner, Messines du Sourbien et de Rouville exposèrent tom a tour les elivers aspects de ees domaines étudies a la Socicle Hydrotechnique de France, on s'at- tachant a démontrer tout l'intérèt de ses travalux et de ses publications.

Enfin N. Ie Président Barrirlon fit une synthèse de ces démonstrations en y assouiant le travail des Commissions et termina par un exposé des diffeultés rencontrées actuellement pour la publication indispensable des communications.

Nous publions ci-après, dans leur intégralité, les allocutions et les exposés faits à cette séance. Elle fut close par la projection du film du Laboratoire Dauphinois d'Hydraulique, Etude sur modèle rédutt des ounrages du port de Zonguldak (Mer Noire), merveilleux documentaire technique gui laissa les nombreux amis de la S.H.F. dans l'enchantement de cetle utile et agréable réunion.

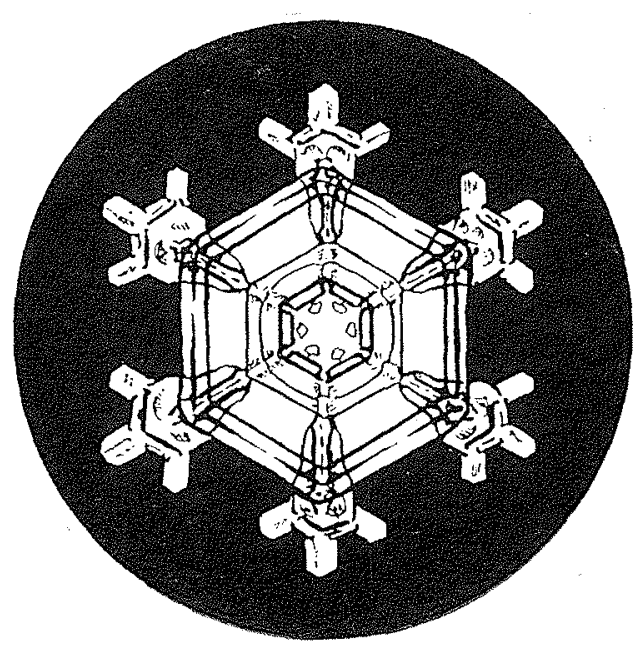

